\title{
Impacto do desmatamento e formação de pastagens sobre a anurofauna de serapilheira em Rondônia
}

\author{
Paulo Sérgio Bernarde ${ }^{1} \&$ Lílian Cristina Macedo ${ }^{2}$
}

\begin{abstract}
1. Laboratório de Herpetologia, Centro Multidisciplinar, Campus Floresta, Universidade Federal do Acre - UFAC, $69980-000$ Cruzeiro do Sul, AC. (snakeBernarde@ hotmail.com)

2. Laboratório Multidisciplinar de Biologia, Faculdade de Educação e Meio Ambiente - FAEMA, Av. Machadinho 4349, Setor 6, 78932 125 Ariquemes, RO. (rhinella@ hotmail.com)
\end{abstract}

\begin{abstract}
The impact of deforestation and pastures on the leaf-litter frog community in Rondônia, Brazil. Abundance and diversity of leaf-litter frog species were studied in forest and pasture in Rondonia, Brazil. Also, the efficiency of use of pitfall traps was examined. Pitfall traps were established in three treatments: forest interior far $(>100 \mathrm{~m})$ from water; forest interior near $(<100 \mathrm{~m})$ water; pasture, far from water. Species richness was typical of the Amazon region, with a total of 1,324 individuals of 27 species in nine families: Aromobatidae (1 species), Brachycephalidae (2), Bufonidae (4), Cycloramphidae (1), Dendrobatidae (1), Hylidae (5), Leiuperidae (1), Leptodactylidae (7) e Microhylidae (5). Pitfall traps captured 57\% of the species known for this location, capturing mainly terrestrial and fossorial species. The greatest richness and diversity of captures were during the rainy season in the forested sites. Forest converted to pasture affects several features of the habitat that reduce habitat quality with respect to these amphibians, including fewer places for reproduction, reduced food supply, loss of leaf-litter and soil compaction all with their influence on microclimate, as well as the arrival of other species more common in open areas.
\end{abstract}

KEYWORDS. Amphibia, Anura, leaf litter, southwestern Amazonia, Rondônia.

RESUMO. A riqueza e a diversidade de anfíbios anuros de serapilheira em áreas de floresta e de pastagem foram estudadas em Rondônia. A eficiência da utilização de armadilhas de interceptação e queda foi analisada. As armadilhas foram instaladas em três ambientes distintos: interior de floresta, distante de ambientes aquáticos permanentes; interior de floresta, a cerca de 100 metros de rio; área de pastagem, distante de ambientes aquáticos permanentes. Foram capturados 1.324 espécimes pertencentes a 27 espécies, distribuídas em nove famílias: Aromobatidae (1 espécie), Brachycephalidae (2), Bufonidae (4), Cycloramphidae (1), Dendrobatidae (1), Hylidae (5), Leiuperidae (1), Leptodactylidae (7) e Microhylidae (5). O emprego de armadilhas de interceptação e queda permitiu registrar 57\% das espécies conhecidas para essa localidade, sendo particularmente eficiente para a captura de anuros de hábitos terrícolas e fossoriais. Foram capturados menos indivíduos nos três meses mais secos e houve maior diversidade durante a estação chuvosa. Maior riqueza e diversidade foram registradas nos ambientes florestados, em relação à pastagem, observando-se decréscimo de espécies com o desmatamento. A conversão da floresta em pastagens ocasiona modificações no ambiente (diminuição de locais para reprodução, diminuição da disponibilidade de alimento, perda da serapilheira, compactação do solo, alterações microclimáticas, aumento da abundância de espécies de áreas abertas, etc.) criando condições desfavoráveis para a sobrevivência de algumas espécies.

PALAVRAS-CHAVE. Amphibia, Anura, serapilheira, sudoeste da Amazônia, Rondônia.

Anfíbios anuros são importantes componentes da fauna de serapilheira nas florestas tropicais (e.g., ALLMON, 1991; GASCON, 1996), servindo de alimento para vários grupos animais (Duellman, 1990; Duellman \& Trueb, 1994) e predando principalmente artrópodos (VITT \& Caldwell, 1994; Lima, 1998). Os poucos estudos realizados na região Neotropical envolvendo anuros de serapilheira utilizaram, para amostragem, o método de parcelas (plots) (e.g., Allmon, 1991; Gascon, 1996; GiaRETTA et al., 1997) ou procura em transectos (e.g., Moreira \& LiMA, 1991; LiMA, 1998).

São escassos os estudos desenvolvidos com anfíbios anuros no Estado de Rondônia, no Sudoeste da Amazônia. Os estudos existentes limitam-se a alguns inventários de espécies (VANZOLInI, 1986; Bernarde et al., 1999; BRANDÃo, 2002), descrição de novas espécies (e.g., Bokermann, 1962; Giaretta et al., 2000) e dieta da herpetofauna de serapilheira (VITT \& CALDWELL, 1994). BERNARDE (2007) registrou os ambientes de reprodução e a temporada de vocalização das espécies de anuros em uma localidade em Espigão do Oeste. No Brasil, existem poucos estudos sobre a anurofauna de serapilheira que utilizaram armadilhas de interceptação e queda para amostragem (ver CECHIN \& MARTINS, 2000; ROCHA et al., 2004; LoEBMANN \& VIEIRA, 2005; BRASILEIRO et al., 2005). Neste trabalho, estudou-se a riqueza e diversidade de anfíbios anuros em áreas de floresta e de pastagem em Rondônia e analisou-se a eficiência da utilização de armadilhas de interceptação e queda (pitfall traps with drift fences) na amostragem.

\section{MATERIAL E MÉTODOS}

A área de estudo compreende a Fazenda Jaburi $\left(11^{\circ} 35^{\prime}-11^{\circ} 38\right.$ ' $\left.\mathrm{S} ; 60^{\circ} 41^{\prime}-60^{\circ} 45^{\prime} \mathrm{W}\right)$, situada no Município de Espigão do Oeste, Rondônia, Brasil (Fig. 1). Situada a cerca de $280 \mathrm{~m}$ de altitude, apresenta média pluviométrica anual de $2.300 \mathrm{~mm}$, com um período de seca que vai de abril a setembro. A precipitação acumulada nesse período foi de $456 \mathrm{~mm}$, enquanto nos meses mais chuvosos (outubro a março) foi de $1.850 \mathrm{~mm}$. A temperatura média anual é de $26^{\circ} \mathrm{C}$, sendo a mínima de $20^{\circ} \mathrm{C}$ e a máxima de $38^{\circ} \mathrm{C}$. Os dados climáticos foram fornecidos pela Prefeitura Municipal de Espigão do Oeste (RO). A Fazenda Jaburi 
apresenta 4.000 ha, sendo 50\% de floresta amazônica ("Mata de Terra Firme") e o restante, áreas de pastagem com predomínio de Brachiaria brizantha (Poaceae). A vegetação local enquadra-se dentro do Domínio Equatorial Amazônico (sensu Ab'Saber, 1977), correspondendo a Floresta Ombrófila Aberta Submontana (Oliveira, 2002). O desmatamento para formação da área de pastagem ocorreu em 1976.

Para a amostragem foi utilizado o método de armadilhas de interceptação e queda, seguindo os procedimentos em HeYER et al. (1994) e as recomendações em CECHIN \& MARTINs (2000). Foram utilizados tambores plásticos (200 1 de capacidade e $90 \mathrm{~cm}$ de altura), enterrados a cada $10 \mathrm{~m}$ e com as aberturas interceptadas por cerca-guia de lona plástica, com $1 \mathrm{~m}$ de altura. Foram instaladas seis séries de armadilhas em linha reta. Cada série consistia de quatro tambores, interligados por cercas que totalizavam $44 \mathrm{~m}$ em cada série. As armadilhas de interceptação e queda foram instaladas em três ambientes distintos (distância mínima de 1.800 m entre eles), com duas séries em cada: (1) dentro de floresta, distante de ambientes aquáticos permanentes; (2) dentro de floresta, a cerca de $100 \mathrm{~m}$ de rio (cerca de $4 \mathrm{~m}$ de largura e $0,80 \mathrm{~m}$ de profundidade) e (3) em área de pastagem, distante de ambientes aquáticos permanentes. Em cada ambiente, as séries de armadilhas foram instaladas à distância de aproximadamente $200 \mathrm{~m}$ entre si. As armadilhas permaneceram abertas ininterruptamente durante um ano ( $8760 \mathrm{~h}$ por balde) e foram monitoradas no máximo a cada dois dias.

A identificação dos espécimes foi feita com base em descrições existentes para espécies do grupo, pertencentes a diferentes localidades na Amazônia (Duellman, 1978; Rodríguez \& Duellman, 1994; De LA Riva et al., 2000). A maioria dos espécimes foi solto após a captura. Espécimes testemunho foram depositados nas seguintes coleções herpetológicas: $\mathrm{CFBH}$, Universidade Estadual Paulista, UNESP, Rio Claro, SP; AAG-UFU, Universidade Federal de Uberlândia, MG; UFAC, Universidade Federal do Acre, Rio Branco, AC. Relação de espécimes testemunho: Allobates aff. marchesianus: CFBH 5135-5139; UFAC 3272, 3277. Pristimantis spp.: CFBH 5142-5155; UFAC 3223, 3248, 3269, 3283, 3305. Chaunus schneideri Werner, 1894: CFBH 5125. Rhaebo

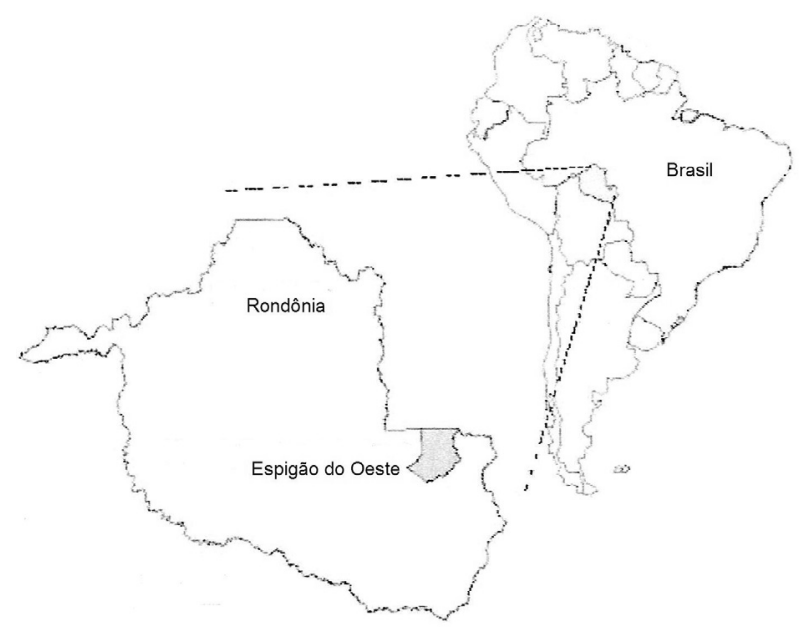

Fig. 1. Localização do Município de Espigão do Oeste em Rondônia. guttatus (Schneider, 1799): CFBH 5124; UFAC 3295-3297. Rhinella margaritifer (Laurenti, 1768): CFBH 5126-5128; UFAC 3224, 3238, 3278, 3279, 3287, 3300. Proceratophrys concavitympanum Giaretta, Bernarde \& Kokubum, 2000: CFBH 5140, 5141; UFAC 3259, 3263, 3264, 3271- 3274, 3280, 3289, 3291, 3306-3308, 3312, 3315, 3316, 3319, 33233328, 3332. Adelphobates quinquevittatus (Steindachner, 1864): UFAC 3292, 3303. Engystomops petersi (Jiménezde-la-Espada, 1872): CFBH 5119-5122; UFAC 3226-3228, 3231-3234, 3239-3241, 3243, 3244, 3252, 3253, 3258, 3261, 3262, 3284, 3285. Leptodactylus andreae (Müller, 1923): CFBH 5159-5169, 5172, 5175, 5176; UFAC 3225, 3237, 3242, 3245, 3246, 3251, 3254-3257, 3260, 3286, 3314. Leptodactylus fuscus (Schneider, 1799): CFBH 5123; UFAC 3270. Leptodactylus lineatus (Schneider, 1799): AAG-UFU 2742-2845. Leptodactylus mystaceus (Spix, 1824): CFBH 5117, 5118; UFAC 3311. Leptodactylus pentadactylus (Laurenti, 1768): CFBH 5113, 5114. Leptodactylus rhodomystax Boulenger, 1884: CFBH 5115, 5116; UFAC 3236, 3266, 3329. Leptodactylus (Lithodytes) sp.: CFBH 5170, 5171, 5173, 5174, 5177, 5188, 5189. Hypsiboas geographicus (Spix, 1824): UFAC 3281. Phyllomedusa tomopterna (Cope, 1868): UFAC 3293. Phyllomedusa vaillanti Boulenger, 1882: UFAC 3294. Scinax ruber (Laurenti, 1768): CFBH 5108. Trachycephalus resinifictrix (Goeldi, 1907): UFAC 3301. Chiasmocleis bassleri Dunn, 1949: CFBH 5134. Chiasmocleis avilapiresae (Andersson, 1945): CFBH 5132, 5133; UFAC 3235, 3268, 3309, 3321. Ctenophryne geayi Mocquard, 1904: CFBH 5129, 5130; UFAC 3247, 3318, 3322. Elachistocleis ovalis (Schneider, 1799) : CFBH 5156-5188; UFAC 3229, 3230, 3249, 3250, 3265, 3276, 3310. Hamptophryne boliviana (Parker, 1927): CFBH 5131.

Para comparação da diversidade de espécies entre os diferentes ambientes e entre as estações chuvosa e seca utilizou-se o Índice de Shannon-Wiener, conforme Magurran (1988). Utilizou-se o teste de Spearman (ZAR, 1984) para verificar possível correlação entre a frequiência de captura de espécimes e de espécies com a pluviosidade ao longo do ano.

\section{RESULTADOS E DISCUSSÃO}

Foram capturados 1.324 espécimes pertencentes a 27 espécies (Tab. I), distribuídas em nove famílias: Aromobatidae (1 espécie), Brachycephalidae (2), Bufonidae (4), Cycloramphidae (1), Dendrobatidae (1), Hylidae (5), Leiuperidae (1), Leptodactylidae (8) e Microhylidae (5). Duas espécies morfologicamente semelhantes, Pristimantis fenestratus (Steindachner, 1864) e Pristimantis sp., foram tratadas juntas em relação à abundância relativa. $\mathrm{O}$ número de espécies amostrado corresponde a $57,4 \%$ do total (47 espécies) conhecido para a localidade (BERnARde, 2007; Tab. II). O método de armadilhas de interceptação e queda é mais eficiente para amostragem de anuros pertencentes a famílias de hábitos terrícolas e fossoriais (Aromobatidae, Brachycephalidae, Bufonidae, Cycloramphidae, Dendrobatidae, Leiuperidae, Leptodactylidae e Microhylidae) do que arborícolas (Centrolenidae e Hylidae) (Tab. II). As cinco espécies de hilídeos registradas não são habitantes freqüentes de chão (ver Duellman, 1990; Allmon, 1991), sendo seu 
Tabela I. Relação das espécies de anuros capturadas nas armadilhas de interceptação e queda na Fazenda Jaburi em Espigão do Oeste (RO) com a respectiva riqueza, número de espécimes e índice de diversidade de Shannon-Wiener (H') para cada unidade amostral. FLO, interior de floresta, distante de ambientes aquáticos; RIO, interior de floresta, próximo a rio; PAS, área de pastagem.

\begin{tabular}{|c|c|c|c|}
\hline FAMÍLIA/ESPÉCIE & FLO & RIO & PAS \\
\hline \multicolumn{4}{|l|}{ AROMOBATIDAE } \\
\hline Allobates aff. marchesianus & & 6 & \\
\hline \multicolumn{4}{|l|}{ BRACHYCEPHALIDAE } \\
\hline Pristimantis spp. & 123 & 78 & 20 \\
\hline \multicolumn{4}{|l|}{ BUFONIDAE } \\
\hline Chaunus marinus (Linnaeus, 1758) & 3 & 6 & \\
\hline Chaunus schneideri Werner, 1894 & & & 8 \\
\hline Rhaebo guttatus (Schneider, 1799) & 1 & 1 & \\
\hline Rhinella margaritifer (Laurenti, 1768) & 19 & 35 & 1 \\
\hline \multicolumn{4}{|l|}{ CYCLORAMPHIDAE } \\
\hline Proceratophrys concavitympanum Giaretta, Bernarde \& Kokubum, 2000 & 25 & 1 & \\
\hline \multicolumn{4}{|l|}{ DENDROBATIDAE } \\
\hline Adelphobates quinquevittatus (Steindachner, 1864) & 2 & 1 & \\
\hline \multicolumn{4}{|l|}{ LEIUPERIDAE } \\
\hline Engystomops petersi (Jiménez-de-la-Espada, 1872) & 118 & 151 & 1 \\
\hline \multicolumn{4}{|l|}{ LEPTODACTYLIDAE } \\
\hline Leptodactylus andreae (Müller, 1923) & 88 & 168 & 72 \\
\hline Leptodactylus fuscus (Schneider, 1799) & & 2 & 16 \\
\hline Leptodactylus lineatus (Schneider, 1799) & 28 & 84 & \\
\hline Leptodactylus mystaceus (Spix, 1824) & 18 & 4 & \\
\hline Leptodactylus pentadactylus (Laurenti, 1768) & 8 & 2 & 2 \\
\hline Leptodactylus rhodomystax Boulenger, 1884 & 56 & 9 & \\
\hline Leptodactylus (Lithodytes) sp. & 10 & 4 & \\
\hline \multicolumn{4}{|l|}{ HYLIDAE } \\
\hline Hypsiboas geographicus (Spix, 1824) & 1 & & \\
\hline Phyllomedusa tomopterna (Cope, 1868) & 2 & & \\
\hline Phyllomedusa vaillanti Boulenger, 1882 & 1 & & \\
\hline Scinax ruber (Laurenti, 1768) & & & 20 \\
\hline Trachycephalus resinifictrix (Goeldi, 1907) & 1 & & \\
\hline \multicolumn{4}{|l|}{ MICROHYLIDAE } \\
\hline Chiasmocleis bassleri Dunn, 1949 & 1 & & \\
\hline Chiasmocleis avilapiresae Peloso \& Sturaro, 2008 & 15 & 11 & \\
\hline Ctenophryne geayi Mocquard, 1904 & 8 & 19 & \\
\hline Elachistocleis ovalis (Schneider, 1799) & 6 & 3 & 64 \\
\hline Hamptophryne boliviana (Parker, 1927) & 1 & & \\
\hline Riqueza & 22 & 18 & 09 \\
\hline Número de espécimes & 535 & 585 & 204 \\
\hline $\mathrm{H}^{\prime}$ & 2,22 & 1,93 & 1,61 \\
\hline
\end{tabular}

registro decorrente de capturas acidentais. No primeiro mês foram registradas 15 espécies e a partir do décimo mês, não houve mais acréscimo de espécies (Fig. 2).

A taxa de captura de anuros nesse estudo foi de 4,6 indivíduos/recipiente/mês. Essa taxa foi maior nos ambientes de floresta (floresta distante de ambientes aquáticos $=5,57$; floresta próxima a rio $=6,09$ ) do que na pastagem $(2,12)$. Esta taxa de captura foi maior do que as obtidas em outros estudos no Brasil (CECHIN \& MARTINS, 2000), com exceção do Cerrado de Itirapina (SP) onde fora apresentado apenas os dados de captura durante a estação chuvosa. Essas diferenças nas taxas de captura podem estar associadas ao tamanho dos recipientes e à quantidade de dias em que as armadilhas permaneceram abertas (ver CECHIN \& MARTINS, 2000). Este método é eficiente em ambientes de floresta e de cerrado (CECHIN \& MARTINS, 2000), porém foi considerado ineficiente para a restinga (RochA et al., 2004).
A utilização de armadilhas de interceptação e queda em estudos de comunidades de anuros possibilita o registro de espécies que raramente são encontradas quando outros métodos empregados são utilizados (CAmpbell \& Christman, 1982). Seis espécies nessa localidade foram registradas exclusivamente por esse método ( $C$. bassleri, C. avilapiresae, C. geayi, A. quinquevittatus, L. mystaceus e L. rhodomystax), não tendo sido registradas pela procura visual e auditiva nos ambientes de reprodução por BERNARDE (2007).

Dos 1.324 espécimes capturados, 1.088 (82,2\%) pertenciam a espécies das famílias Brachycephalidae, Cycloramphidae, Leiuperidae e Leptodactylidae (Tab. II) e $976(73,7 \%)$ corresponderam a apenas sete espécies: $L$. andreae, Pristimantis spp., L. lineatus, E. petersi, E. ovalis e L. rhodomystax (Fig. 3). Leptodactylus lineatus, tida como espécie pouco amostrada em outros estudos (e.g. DuELlMAN, 1978), foi uma das mais abundantes utilizandose as armadilhas de interceptação e queda (Tab. III). 
Tabela II. Total de espécies previamente conhecidas para a Fazenda Jaburi, Espigão do Oeste, (RO) (BERNARDE, 2007) e o número de espécies e espécimes registrados por família mediante o uso de armadilhas de interceptação e queda. Proporção entre parênteses.

\begin{tabular}{lccc}
\hline FAMÍLIA & Espécies conhecidas & Espécies registradas & Espécimes \\
Aromobatidae & 1 & $1(100 \%)$ & $6(0,4 \%)$ \\
Brachycephalidae & 2 & $2(100 \%)$ & $221(16,7 \%)$ \\
Bufonidae & 4 & $4(100 \%)$ & $74(5,6 \%)$ \\
Centrolenidae & 1 & $0(0 \%)$ & $0(0 \%)$ \\
Cycloramphidae & 1 & $1(100 \%)$ & $26(1,9 \%)$ \\
Dendrobatidae & 1 & $1(100 \%)$ & $3(0,2 \%)$ \\
Hylidae & 23 & $5(21,7 \%)$ & $25(1,9 \%)$ \\
Leiuperidae & 1 & $1(100 \%)$ & $270(20,4 \%)$ \\
Leptodactylidae & 8 & $7(87,5 \%)$ & $571(43,1 \%)$ \\
Microhylidae & 5 & $5(100 \%)$ & $128(9,7 \%)$ \\
\hline Total & 47 & 27 & 1.324 \\
\hline
\end{tabular}

Tabela III. Quantidade de anuros capturados mensalmente em armadilhas de interceptação e queda na Fazenda Jaburi, Espigão do Oeste (RO) entre abril de 2001 a março de 2002 e total das capturas, da pluviosidade e do índice de diversidade (H') referentes aos meses secos e chuvosos. Espécies conforme Tabela I.

\begin{tabular}{|c|c|c|c|c|c|c|c|c|c|c|c|c|c|c|}
\hline \multirow[b]{2}{*}{ Espécie / Meses } & \multicolumn{7}{|c|}{ Meses secos } & \multicolumn{7}{|c|}{ Meses chuvosos } \\
\hline & A & M & $\mathrm{J}$ & $\mathrm{J}$ & A & $S$ & $\Sigma$ & $\mathrm{O}$ & $\mathrm{N}$ & $\mathrm{D}$ & $\mathrm{J}$ & $\mathrm{F}$ & M & $\Sigma$ \\
\hline A. aff. marchesianus & 2 & 2 & & & & & 4 & & 1 & & 1 & & & 2 \\
\hline Pristimantis spp. & 23 & 51 & 21 & 34 & 44 & 19 & 192 & 9 & 1 & & 5 & 5 & 9 & 29 \\
\hline C. marinus & & & & & & & & 1 & 1 & 1 & 5 & 1 & & 9 \\
\hline C. schneideri & & & & & & & & & 2 & 2 & & 3 & 1 & 8 \\
\hline R. guttatus & & & & & & & & & 2 & & & & & 2 \\
\hline R. margaritifer & 3 & 5 & 1 & 5 & & 2 & 16 & 5 & 11 & 4 & 6 & 5 & 8 & 39 \\
\hline P. concavitympanum & 1 & & & & & & 1 & 5 & 3 & 3 & 6 & 4 & 4 & 25 \\
\hline A. quinquevittatus & & & 1 & & & 1 & 2 & 1 & & & & & & 1 \\
\hline H. geographicus & & & 1 & & & & 1 & & & & & & & \\
\hline P. tomopterna & & & & & & & & & & & 2 & & & 2 \\
\hline P. vaillanti & & & & & & 1 & 1 & & & & & & & \\
\hline S. ruber & & & & & & & & & & 9 & 2 & 3 & 6 & 20 \\
\hline T. resinifictrix & & & & 1 & & & 1 & & & & & & & \\
\hline E. petersi & 43 & 41 & 10 & 4 & 2 & 19 & 119 & 11 & 24 & 21 & 11 & 51 & 33 & 151 \\
\hline L. andreae & 52 & 63 & 12 & 8 & 2 & 74 & 211 & 47 & 5 & 9 & 9 & 31 & 16 & 117 \\
\hline L. fuscus & 3 & 1 & & & & 7 & 11 & 1 & 2 & & & & 4 & 7 \\
\hline L. lineatus & 16 & 10 & 2 & & & 10 & 38 & 18 & 20 & 8 & 4 & 10 & 14 & 74 \\
\hline L. mystaceus & 1 & & 1 & & & & 2 & 4 & 9 & 3 & 3 & & 1 & 20 \\
\hline L. pentadactylus & 2 & & & & & & 2 & 2 & 3 & 2 & 3 & & & 10 \\
\hline L. rhodomystax & 3 & & & & & & 3 & 7 & 24 & 12 & 16 & 2 & 1 & 62 \\
\hline Leptodactylus sp. & 1 & 3 & 1 & & 1 & 3 & 10 & 4 & 1 & & & & & 4 \\
\hline C. bassleri & & & & & & & & & 1 & & & & & 1 \\
\hline C. avilapiresae & 6 & 1 & & & & 1 & 8 & 5 & 7 & 2 & 3 & 1 & & 18 \\
\hline C. geayi & 1 & 1 & & & & 1 & 3 & 3 & 7 & 2 & 9 & 1 & 2 & 24 \\
\hline E. ovalis & 10 & 8 & & & & 5 & 23 & 7 & 17 & 4 & & 5 & 17 & 50 \\
\hline H. boliviana & & & & & & & & & 1 & & & & & 1 \\
\hline$\Sigma$ espécimes & 167 & 186 & 50 & 52 & 49 & 143 & 648 & 130 & 142 & 82 & 85 & 122 & 116 & 676 \\
\hline$\Sigma$ espécies & 15 & 11 & 9 & 5 & 4 & 12 & 19 & 16 & 20 & 14 & 15 & 13 & 13 & 23 \\
\hline Pluviosidade (mm) & 94 & 117 & 41 & 0 & 0 & 204 & & 174 & 240 & 370 & 382 & 333 & 351 & \\
\hline $\mathrm{H}^{\prime}$ & & & & & & & 1,77 & & & & & & & 2,46 \\
\hline
\end{tabular}

Nos ambientes florestados foram registradas maiores riquezas (22 e 18 espécies) e índices de diversidade $\left(H^{\prime}=2,26 ; H^{\prime}=1,93\right)$ em relação à área de pastagem (9 espécies; $H^{\prime}=1,61$ ) (Tab. I). A conversão da floresta em pastagens ocasiona modificações no ambiente, como diminuição de locais para reprodução, diminuição da disponibilidade de alimento, perda da serapilheira, compactação do solo, alterações microclimáticas, aumento da abundância de espécies de áreas abertas, etc. (Aichinger, 1991; Alves et al., 1999;
TAPIA-CoRAl et al., 1999; VITT \& CALDWELl, 2001), criando condições desfavoráveis para a sobrevivência de algumas espécies. A diminuição da diversidade de anuros devido à transformação de florestas em áreas de pastagens e de lavouras foi observada, também, em outras regiões (Aichinger, 1991; HeINEN, 1992; TOCHER, 1998; Vallan, 2002). Três espécies (C. shneideri, $E$. ovalis e L. fuscus) encontradas exclusivamente ou preferencialmente no ambiente de pastagem utilizam preferencialmente ambientes abertos durante atividade 
reprodutiva e outras duas (L. andreae e P. fenestratus) que ocorreram em todos ambientes utilizam tanto áreas de florestas como abertas (DuELLMAN, 1978; BERNARDE, 2007).

Não houve correlação significativa entre o número de espécimes capturados e a pluviosidade (Fig. 4; $\mathrm{r}=0,2551, \mathrm{p}=0,449)$, porém houve em relação ao número de espécies $(\mathrm{r}=0,7306, \mathrm{p}=0,01)$. Entretanto, foram capturados menos indivíduos nos meses mais secos (junho a agosto) (Fig. 4, Tab. III). Apesar de ter sido registrada maior diversidade nos meses mais chuvosos $\left(H^{\prime}=2,46\right)$ em relação aos secos $\left(H^{\prime}=1,77\right)$ (Tab. III), essa diferença não foi estatisticamente significativa $(\mathrm{t}=0,258$, $\mathrm{p}=0,6)$. A maior captura de anuros na estação chuvosa deve estar associada com a temporada de vocalização da maioria das espécies que ocorre nesse período (BERNARDE,

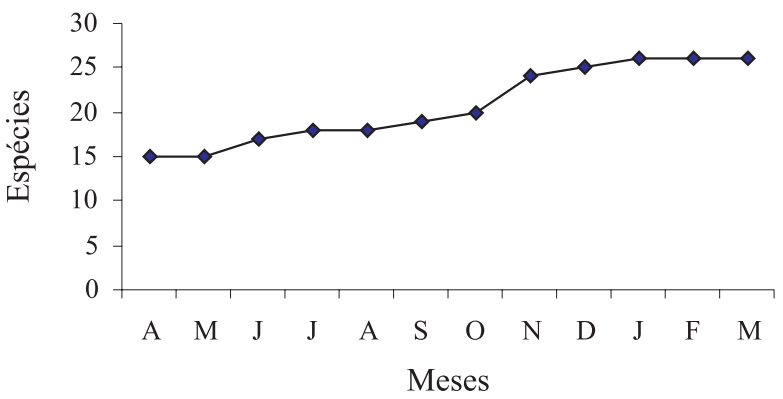

Fig. 2. Curva cumulativa de espécies registradas na Fazenda Jaburi, Espigão do Oeste (RO) entre os meses de abril de 2001 a março de 2002, mediante o uso de armadilhas de interceptação e queda.

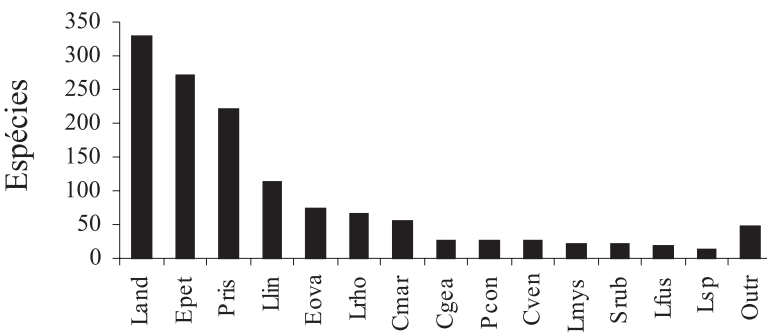

Fig. 3. Abundância relativa das espécies de anuros capturadas nas armadilhas de interceptação e queda na Fazenda Jaburi, Espigão do Oeste (RO). Siglas correspondem à primeira letra do gênero e as três primeiras da espécie, conforme Tabela I. Exceção: Lsp, Leptodactylus (Lithodytes) sp.; Pris, Pristimantis spp.; Outr, outras espécies.

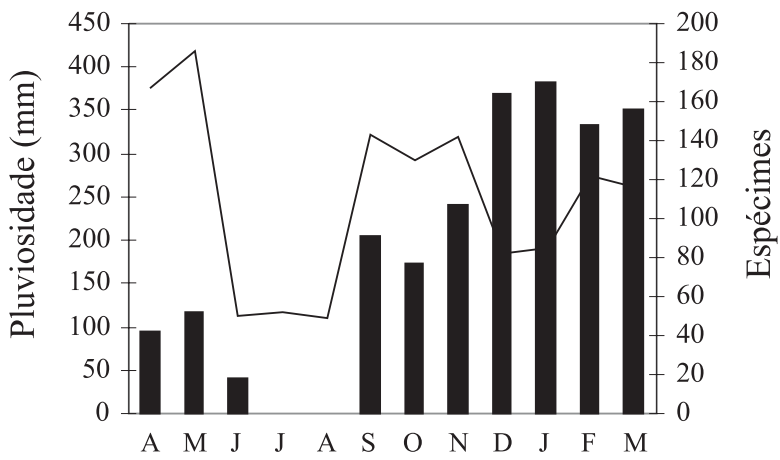

Fig. 4. Número de capturas de anuros (linha) e pluviosidade (barras) na Fazenda Jaburi, Espigão do Oeste (RO), entre os meses de abril de 2001 a março de 2002.
2007) e o respectivo deslocamento de indivíduos machos para os ambientes de reprodução. Algumas espécies ocorreram preferencialmente nos meses mais chuvosos, como $L$. rhodomystax, $P$. concavitympanum e os microhilídeos, enquanto outras, como L. andreae e Pristimantis spp., nos meses mais secos.

Agradecimentos. Aos colegas Célio Haddad, Marcelo Kokubum, Moisés Barbosa de Souza e Reginaldo A. Machado pela ajuda em algumas fases desse trabalho. Aos proprietários da Fazenda Jaburi Eduardo Garcia, Celso Garcia e família, pelo apoio logístico. A Carlos Alberto Bernarde e família pelo apoio logístico em Espigão do Oeste. Ao Centro de Conservação e Manejo de Répteis e Anfíbios - RAN, do IBAMA, pelas licenças de coleta concedida (Processo 02001.006649/00-60; Licenças 246/2000DIFAS/DIREC e 021/02-RAN). À CAPES pela bolsa de estudos concedida (PSB). Ao revisor anônimo da Iheringia pela leitura e sugestões feitas a esse manuscrito.

\section{REFERÊNCIAS BIBLIOGRÁFICAS}

AB' SABER, A. N. 1977. Os domínios morfoclimáticos da América do Sul. Boletim do Instituto de Geografia da Universidade de São Paulo 52:1-21.

Aichinger, M. 1991. Faunal deficit of anurans in tropical farmland of Amazonian Peru. Alytes 9(1):23-32.

Allmon, W. D. 1991. A plot study of forest floor litter frogs, Central Amazon, Brazil. Journal of Tropical Ecology 7:503-522.

Alves, F. S. M.; Fisch, G. \& Vendrame, I. F. 1999. Modificações do microclima e regime hidrológico devido ao desmatamento na Amazônia: estudo de um caso em Rondônia (RO), Brasil. Acta Amazonica 29:395-409.

Bernarde, P. S. 2007. Ambientes e temporada de vocalização da anurofauna no Município de Espigão do Oeste, Rondônia, Sudoeste da Amazônia - Brasil (Amphibia: Anura). Biota Neotropica 7(2):87-92

Bernarde, P. S.; Kokubum, M. C. N.; Machado, R. A. \& Anjos, L. 1999. Uso de habitats naturais e antrópicos pelos anuros em uma localidade no Estado de Rondônia, Brasil (Amphibia: Anura). Acta Amazonica 29(4):555-562.

Bokermann, W. C. 1962. Duas novas espécies de "Hyla" de Rondônia, Brasil. Revista Brasileira de Biologia 23(3):247-250.

BRANDÃo, R. A. 2002. Avaliação ecológica rápida da herpetofauna nas reservas extrativistas de Pedras Negras e Curralinho, Costa Marques, RO. Brasil Florestal 74:61-73.

Brasileiro, C. A.; Sawaya, R. J.; Kiefer, M. C. \& Martins, M. 2005. Amphibians of an open cerrado fragment in southeastern Brazil. Biota Neotropica 5(2):1-17.

Campbell, H. W. \& Christman, S. P. 1982. Field techniques for herpetofaunal community analysis, In: ScotT JR., N. J. ed. Herpetological communities: a Symposium of the Society for the Study of Amphibians and Reptiles and the Herpetologists' League. Washington, U. S. Fish Wildlife Service. p.193-200.

Cechin, S. Z. \& Martins, M. 2000. Eficiência de armadilhas de queda (pitfall traps) em amostragem de anfíbios e répteis no Brasil. Revista Brasileira de Zoologia 17(3):729-740.

De La Riva, I.; Kohler, J.; Lotters, S. \& Reichle, S. 2000. Ten years of research on Bolivian amphibians: Update checklist, distribution, taxonomic problems, literature and iconography. Revista Española de Herpetologia 14:19-164.

Duellman, W. E. 1978. The biology of an equatorial herpetofauna in Amazonian Equador. University of Kansas, Museum of Natural History Miscellaneous Publications 65:1-352. 1990. Herpetofaunas in Neotropical rainforests: comparative composition, history, and resource use. In: Gentry, A. H. ed. Four Neotropical Rainforests. New Haven, Yale University. p. 455-505.

Duellman, W. E. \& Trueb, L. 1994. Biology of Amphibians. Baltimore, The Johns Hopkins University. 670p.

Gascon, C. 1996. Amphibian litter fauna and river barriers in flooded and non-flooded Amazonian Rain Forest. Biotropica 28(1):136-140. 
Giaretta, A. A.; Sawaya, R. J.; Machado, G.; Araújo, M. S.; Facure, K. G.; Medeiros, H. F. \& Nunes, R. 1997. Diversity and abundance of litter frogs at altitudinal sites at Serra do Japi, Southeastern Brazil. Revista Brasileira de Zoologia 14(2):341-346.

Giaretta, A. A.; Bernarde, P. S. \& Kokubum, M. C. N. 2000. A new species of Proceratophrys (Anura: Leptodactylidae) from the Amazon Rain Forest. Journal of Herpetology 34(2):173-178.

Heinen, J. T. 1992. Comparisons of the leaf litter herpetofauna in abandoned cacao plantations and primary rain forest in Costa Rica: some implications for faunal restoration. Biotropica 24(3):431-439.

Heyer, R. H.; Donnelly, M. A.; Mcdiarmid, R. W.; Hayek, L. C. \& Foster, M. S. 1994. Measuring and monitoring biological diversity: Standard methods for amphibians. Washington, Smithsonian Institution. 364p.

LimA, A. P. 1998. The effects of size on the diets of six sympatric species of postmetamorphic litter anurans in Central Amazônia. Journal of Herpetology 32(3):392-399.

Loebmann, D. \& Vieira, J. P. 2005. Relação dos anfíbios do Parque Nacional da Lagoa do Peixe, Rio Grande do Sul, Brasil. Revista Brasileira de Zoologia 22(2):339-341.

Magurran, A. E. 1988. Ecological diversity and its measurement. London, Groon Helm. 179p.

Moreira, G. \& Lima, A. P. 1991. Seasonal patterns of juvenile recruitment and reproduction in four species of leaf litter frogs in Central Amazônia. Herpetologica 47(3):295-300.

Oliveira, O. A. 2002. Geografia de Rondônia - espaço e produção. Porto Velho, Dinâmica.

Rocha, C. F. D.; Van-Sluys, M.; Hatano, F. H.; Boquimpani-Freitas, L.; Marra, R. V. \& Marques, R. V. 2004. Relative efficiency of anuran sampling methods in a restinga habitat (Jurubatiba,
Rio de Janeiro, Brazil). Revista Brasileira de Biologia 64(4):879-884.

Rodriguez, L. O. \& Duellman, W. E. 1994. Guide to the Frogs of the Iquitos Region, Amazonian Peru. Lawrence, Kansas. Asociación de Ecologia y Conservación, Amazon Center for Environmental Education and Research, and Natural History Museum, The University of Kansas. 80p.

Tapia-Coral, S. C.; Luizão, F. J. \& Wandelli, E. V. 1999. Macrofauna da liteira em sistemas agroflorestais sobre pastagens abandonadas na Amazônia Central. Acta Amazonica 29(3):477-495.

Tocher, M. 1998. Diferenças na composição de espécies de sapos entre três tipos de floresta e campo de pastagem na Amazônia central. In: Gascon, C. \& Moutinho, P. eds. Floresta Amazônica: Dinâmica, Regeneração e Manejo. Manaus, Ministério da Tecnologia e Ciência, Instituto de Pesquisas da Amazônia. p.219-232.

VALLAN, D. 2002. Effects of anthropogenic environmental changes on amphibian diversity in the rain forests of eastern Madagascar. Journal of Tropical Ecology 18:725-742.

Vanzolini, P. E. 1986. Levantamento herpetológico da área do Estado de Rondônia sob a influência da rodovia Br-364. Polonoreste/Ecologia Animal. Relatório de Pesquisa $\mathbf{n}^{\mathbf{0} 1}$, Brasília, CNPq. 50p.

VitT, L. V. \& Caldwell, J. P. 1994. Resource utilization and guild structure of small vertebrates in the Amazon forest leaf litter. Journal of Zoology 234(4):463-476.

. 2001. The effects of logging on reptiles and amphibians of tropical forests. In: Fimbel, R. A.; Grajal, A. \& Robinson, J. G. eds. The Cutting Edge: Conserving Wildlife in Logged Tropical Forests. New York, Columbia University. p.239-259.

ZAR, J. H. 1984. Biostatistical analysis. New Jersey, PrenticeHall International, 2.ed. 718p.

Recebido em março de 2006. Aceito em novembro de 2008. ISSN 0073-4721

Artigo disponível em: www.scielo.br/isz 